\title{
TORIC DEGENERATIONS OF GIT QUOTIENTS, CHOW QUOTIENTS, AND $\bar{M}_{0, N}{ }^{*}$
}

\author{
YI $\mathrm{HU}^{\dagger}$
}

Key words. Toric Degeneration, chow quotient, moduli of curves.

AMS subject classifications. $14 \mathrm{Dxx}, 14 \mathrm{Exx}$

1. Introduction. The moduli space $\bar{M}_{0, n}$ plays important roles in algebraic geometry and theoretical physics. Yet, some basic properties of $\bar{M}_{0, n}$ still remain open. For example, $\bar{M}_{0, n}$ is rational and nearly toric (that is, it contains a toric variety as a Zariski open subset), but it is not a toric variety itself starting from dimension $2(n \geq 5)$. So, a basic question is: Can it be degenerated flatly to a projective toric variety? Finding toric degeneration is important because many calculations may then be done in terms of combinatorial data extracted out of polytopes and/or fans. The main purpose of this note is to answer the above question in affirmative.

We achieve this by showing that the Chow quotient of the Grassmannian $\operatorname{Gr}\left(2, \mathbb{C}^{n}\right)$ admits toric degeneration, which in turn, follows from a theorem that we prove for toric degenerations of more general Chow quotients. Consequently, this implies as well that the Chow quotients of higher Grassmannians ([19], [26], [25]), hence also (the main component of) the moduli spaces of hyperplane arrangements ([11]) and (the main component of) the moduli spaces of semi log canonical pairs ([1], [2], [3], [10], [22]), can be degenerated flatly to projective toric varieties. Along the way, we also argued that GIT quotient of a flat family is again flat. In particular, all GIT quotients of flag varieties by maximal tori can be flatly degenerated to projective toric varieties ([9] and [12]).

\section{Flat family of GIT quotients.}

2.1. Flat family of $G$-varieties. Throughout the paper, we will work over the field of complex numbers. Let $f: Y \rightarrow B$ be a flat family of projective varieties. Assume that a reductive algebraic group $G$ acts algebraically on the family and preserves fibers. That is, $G$ acts on the total space $Y$, acts on the base trivially, and $f$ is $G$-equivariant.

Let $L$ be any $G$-linearized ample line bundle over $Y$ and $L_{t}$ be the restriction of $L$ to $Y_{t}=f^{-1}(t)$ with the induced linearization for any $t \in B$. By [28], we have

$$
Y_{t}^{s s}\left(L_{t}\right)=Y^{s s}(L) \cap Y_{t}
$$

and

$$
Y_{t}^{s}\left(L_{t}\right)=Y^{s}(L) \cap Y_{t}
$$

Using these identities, the assumption that $G$ acts trivially on $B$, and $f$ is $G$ equivariant, we obtain an induced map

$$
\underline{f}: Y^{s s}(L) / / G \rightarrow B
$$

\footnotetext{
* Received July 29, 2006; accepted for publication April 11, 2007.

${ }^{\dagger}$ Department of Mathematics, University of Arizona, Tucson, AZ 86721, USA (yhu@math.arizona. edu).
} 
with fibers $Y_{t}^{s s}\left(L_{t}\right) / / G$ for any $t \in B$.

When $B$ is regular and of dimension 1, the flatness of $f$ is automatic. We will show that $\underline{f}$ is always flat provided that the base scheme $B \overline{\text { is integral. }}$

Theorem 2.1.1. Assume that the base scheme $B$ is integral. Then the induced family $\underline{f}: Y^{s s}(L) / / G \rightarrow B$ of GIT quotients $Y_{t}^{s s}\left(L_{t}\right) / / G(t \in B)$ is always flat.

Proof. Replace $L$ by a large tensor power, we may assume that $L$ is very ample and descends to a very ample line bundle $M$ over the quotient $Y^{s s}(L) / / G$. Hence for every $n>0, L^{n}$ descends to the line bundle $M^{n}$ over $Y^{s s}(L) / / G$. It follows that $L_{t}^{n}$ descends to $M_{t}^{n}$ for all $t \in B$ simultaneously. Now, by the Theorem of "Quantization commutes with Reduction" (see, for example, [30]), we have that for $n>>0$,

$$
H^{0}\left(Y_{t}^{s s}\left(L_{t}\right) / / G, M_{t}^{n}\right)=H^{0}\left(Y_{t}, L_{t}^{n}\right)^{G} .
$$

This shows that $\operatorname{dim} H^{0}\left(Y_{t}^{s s}\left(L_{t}\right) / / G, M_{t}^{n}\right)$ is independent of the parameter $t$ because $\operatorname{dim} H^{0}\left(Y_{t}, L_{t}^{n}\right)^{G}$ is so. Hence $\underline{f}: Y^{s s}(L) / / G \rightarrow B$ is flat.

\subsection{Toric setting.}

Toric Setting 2.2.1. Consider again our flat family $f: Y \rightarrow B$ with an integral base $B$. Let $0 \in B$ be a distinguished point such that $Y_{0}$ is a toric variety with the maximal torus $\mathbb{T}$. Assume in addition that $G$ is a subtorus of $\mathbb{T}$ whose action on $Y_{0}$ is induced from that of $\mathbb{T}$.

Under this assumption, $Y_{0}^{s s}\left(L_{t}\right) / / G$ is a toric variety with the maximal torus $\mathbb{T} / G$. Hence we have

Corollary 2.2.2. Under the toric setting, $f: Y^{s s}(L) / / G \rightarrow B$ provides a flat toric degeneration for generic fibers of $\underline{f}$.

Apply the above to toric degenerations of complete and partial flag varieties, we re-obtain a result of Foth and Hu [9] (see also [12] for the case of $\operatorname{Gr}\left(2, \mathbb{C}^{n}\right)$ ).

Corollary 2.2.3. GIT quotients of complete and partial flag varieties by the maximal torus degenerate flatly to projective toric varieties.

We remark here that the degenerations in Theorem 2.1.1, Corollaries 2.2.2 and 2.2.3 all depend on the flat families that we start with.

2.3. Singularities of the family. Suppose we start with a family $f: Y \rightarrow B$ with a nonsingular total space $Y$, suppose we can also find a $G$-linearized ample line bundle $L$ over $Y$ such that $Y^{s s}(L)=Y^{s}(L)$. Then we will also have $Y_{t}^{s s}\left(L_{t}\right)=Y_{t}^{s}\left(L_{t}\right)$ for all $t \in B$. In this case, $Y^{s s}(L) / / G$ has at worst finite quotient singularities, so does every $Y_{t}^{s s}\left(L_{t}\right) / / G$. In other words, $f: Y^{s s}(L) / / G \rightarrow B$ is a flat family of orbifolds with the total space of the family also being an orbifold. This may not be achievable for general group $G$ but is always achievable when $G$ is a torus, for example in the the toric setting.

\section{Flat family of Chow quotients.}

3.1. Family of general Chow quotients. In this section, we assume in addition that the base $B$ is projective. Hence the total variety $Y$ is also projective.

As in [16], we will call the Chow cycles in $Y / /{ }^{c h} G$ Chow fibers. By the assumptions of the action, every Chow fiber is vertical, meaning that it is contained in the fiber $Y_{t}$ for some $t \in B$. This leads to a map

$$
f^{c h}: Y / /^{c h} G \rightarrow B \text {. }
$$


To see that this is a projective morphism. We may treat $Y$ as a scheme over $B$ acted upon by the trivial group scheme $G / B=G \times B$. Use the Chow scheme $\operatorname{Ch}(Y / B)$ ([24]), we can embed the space the closures of generic $(G / B)$ - orbits into $\mathrm{Ch}(Y / B)$ and take closure of the image to get the Chow quotient $(Y / B) / /^{\text {ch }}(G / B)$. As variety, this is the same as $Y / /{ }^{c h} G$. Hence $f^{c h}: Y / /{ }^{c h} G \rightarrow B$ is a projective morphism.

Hence we have

THEOREM 3.1.1. When $B$ is regular and of dimension $1, f^{\text {ch }}$ is a flat family.

We suspect this is always true as long as $f$ is flat. But we do not need this generality for applications.

TheOREm 3.1.2. Let $f: Y \rightarrow B$ be as in Toric Setting 2.2.1. Assume that the base $B$ is regular and of dimension 1. Then $f^{c h}$ is a flat family of the Chow quotients $Y_{t} /{ }^{c h} G(t \in B)$ with the central fiber $Y_{0} / /{ }^{c h} G$ a projective toric variety.

Proof. We need to check that the fiber of $f^{c h}: Y / /^{c h} G \rightarrow B$ over $t \in B$ is exactly $Y_{t} /{ }^{c h} G$. This is certainly true for generic fibers.

Let $\delta$ be the homology class of the closure of generic torus orbit on $Y$ (or on $Y_{t}$ for generic $t$ ). Let $t_{0}$ be any given point in $B$ and $\delta_{0}$ be the homology class of the closure of generic torus orbit on $Y_{t_{0}}$. Consider a family of orbit closures $\left\{Z_{t}\right\}$ over (very) generic points $t$ near $t_{0}$ such that each $Z_{t}$ is a generic orbit closure in $Y_{t}$ (that is, the homology class of $Z_{t}$ is $\delta$ ). Consider the limit of $\left\{Z_{t}\right\}$ as $t$ approaches $t_{0}$, the limit is a sum of orbits closures on $Y_{t_{0}}$ ([19]). If none of the homology classes of the orbit closures in the sum is $\delta_{0}$, then generic orbit closures on $Y_{t_{0}}$ will not appear in the fiber of $f^{c h}$ over $t_{0}$. But, this would imply that the fiber of $f^{c h}$ over $t_{0}$ has wrong (smaller) dimension, a contradiction. So, a generic orbit closures on $Y_{t_{0}}$ should appear in the sum. This implies that either $\delta=\delta_{0}$ or $\delta$ is a constant multiple of $\delta_{0}$. In either case, fiber of $f^{c h}$ over $t_{0}$ can be identified with $Y_{t_{0}} /{ }^{c h} G$.

It remains to show that $Y_{0} / /{ }^{c h} G$ is a projective toric variety, but this follows readily from $[20]$.

REMARK 3.1.3. Unlike the case of GIT quotients where different choices may be available, Chow quotient is unique, and in general, can have very nasty singularities.

3.2. Toric degeneration of Chow quotients of flag varieties. Now consider any flat toric degeneration of a (partial) flag variety, e.g, the Grassmannian $\operatorname{Gr}\left(k, \mathbb{C}^{n}\right)$, over the affine space $\mathbb{C}$, we can easily extend this family into a flat family over $\mathbb{P}^{1}$. Then apply the above discussions to the Chow quotients of this family, we obtain

TheOREM 3.2.1. For every flat degeneration of a (partial) flag variety over $\mathbb{P}^{1}$ as in Toric Setting 2.2.1 such that the group $G$ is the maximal torus, there is an induced flat family over $\mathbb{P}^{1}$ such that generic fibers are isomorphic to the Chow quotient of the flag variety and the central fiber over 0 is a projective toric variety.

Hence, in particular, by applying [27] and the above, we have

Corollary 3.2.2. The Chow quotients of Grassmannians studied by Kapranov ([19]) and later by Lafforgue ([25], [26]) and again by Hacking, Keel and Tevelev ([10], [22], [11]) admit flat toric degenerations. The same holds for the Chow quotients of all (partial) flag varieties.

Isolating the special case of $\operatorname{Gr}\left(2, \mathbb{C}^{n}\right)$, we then have the following important corollary: 
Corollary 3.2.3. For every flat degeneration of $\operatorname{Gr}\left(2, \mathbb{C}^{n}\right)$ over $\mathbb{P}^{1}$ as in Toric Setting 2.2.1 such that the group $G$ is the maximal torus of $\mathrm{PGL}_{n}$, there is an induced flat family over $\mathbb{P}^{1}$ such that generic fibers are isomorphic to $\bar{M}_{0, n}$ and the central fiber over 0 is a projective toric variety $\bar{N}_{0, n}$.

Proof. All we need is to apply Kapranov's theorem that $\bar{M}_{0, n}$ is isomorphic to the Chow quotient of $\operatorname{Gr}\left(2, \mathbb{C}^{n}\right)$ by the maximal torus. $\mathrm{Q}$

REMARK 3.2.4. $\bar{N}_{0, n}$ depends on the family that we start with. In this paper, by abusing notation slightly, we will always use the same notation $\bar{N}_{0, n}$ to denote any toric degeneration of $\bar{M}_{0, n}$ that is being considered.

4. Toric Chow Quotients of Flag Varieties and Toric $\bar{M}_{0, n}$. The results so far obviously apply to: quotients of flag vareities, quotients of Schubert varieties, quotients of the products of Grassmannians ( see [15]), and so on. But we will devote the rest of the paper solely to flag varieties and $\bar{N}_{0, n}$.

4.1. Toric Chow quotients of flag varieties. Let $G$ be a connected complex semisimple group ${ }^{1}, B$ a Borel subgroup, $U$ its unipotent radical, and $H$ a Cartan subgroup such that $B=H U$. Let also $\Phi=\Phi(G, H)$ be the system of roots, $\Phi^{+}=$ $\Phi^{+}(B, H)$ the subset of positive roots, and $\left\{\alpha_{1}, \ldots, \alpha_{r}\right\}$ the basis of simple roots, where $r$ is the rank of $G$. Let $\Lambda$ be the weight lattice of $G$ and $\Lambda^{+}$the subset of dominant weights. For $\lambda \in \Lambda^{+}$we denote by $V(\lambda)$ the irreducible $G$-module with highest weight $\lambda$. Let $P_{\lambda} \supset B$ be the parabolic subgroup of $G$ which stabilizes a highest weight vector in $V(\lambda)$. Also denote by $L_{\lambda}=G \times_{P_{\lambda}} \mathbb{C}$ the $G$-linearized line bundle on $X_{\lambda}:=G / P_{\lambda}$ corresponding to the character $\lambda$ extended to $P_{\lambda}$.

Let $W$ be the Weyl group and $w_{0} \in W$ the longest element of length $\ell$. Choose a reduced decomposition

$$
\underline{w_{0}}=s_{i_{1}} s_{i_{2}} \cdots s_{i_{\ell}}
$$

into a product of simple reflections. The space $A:=\mathbb{C}[G]^{U}$ of regular, right $U$ invariant functions on $G$, has a so-called canonical basis $\left(b_{\lambda, \phi}\right)$, where each $b_{\lambda, \phi}$ is an eigenvector for both left and right $H$-action. For the right $H$-action it has weight $\lambda$ and for the left $H$-action the weight is given by

$$
-\lambda+t_{1} \alpha_{i_{1}}+\cdots+t_{\ell} \alpha_{i_{\ell}}
$$

where $b_{\lambda, \phi}$ is parameterized by

$$
\left(\lambda, t_{1}, \ldots, t_{\ell}\right) \in \Lambda^{+} \times \mathbb{N}^{\ell}
$$

(The parameterizations of) $b_{\lambda, \phi}$ generate a rational convex polyhedral cone

$$
\mathcal{C}_{\underline{w_{0}}} \subset \Lambda_{\mathbb{R}} \times \mathbb{R}^{\ell}
$$

(see, e.g., [6]). Let $p_{1}$ and $p_{2}$ be the projection of $\Lambda_{\mathbb{R}} \times \mathbb{R}^{\ell}$ to the first and second factor, respectively. Then for any fixed $\lambda \in \Lambda^{+}, Q(\lambda)=p_{1}^{-1}(\lambda) \cap \mathcal{C}_{w_{0}}$ is the so-called string

\footnotetext{
${ }^{1}$ We note here that this group $G$ is different than the one used in all the previous sections. For example, the group $G$ in Toric Setting 2.2 .1 will be replaced instead by a Cartan subgroup $H$ in this section. We apologize for any possible confusion this may cause.
} 
polytope of $\lambda . Q(\lambda)$ may be identified with its image in $\mathbb{R}^{\ell}$ via the second projection $p_{2}: \Lambda_{\mathbb{R}} \times \mathbb{R}^{\ell} \rightarrow \mathbb{R}^{\ell}$. Let

$$
\pi_{\lambda}: \mathbb{R}^{\ell} \rightarrow \Lambda_{\mathbb{R}}
$$

be defined by

$$
\left(t_{1}, \ldots, t_{\ell}\right) \mapsto-\lambda+t_{1} \alpha_{i_{1}}+\cdots+t_{\ell} \alpha_{i_{\ell}} .
$$

Then $\pi_{\lambda}$ sends the string polytope $Q(\lambda)$ onto the convex hull of the Weyl group orbit of the dual weight $\lambda^{*}=-w_{0} \cdot \lambda$ :

$$
\pi_{\lambda}(Q(\lambda))=\operatorname{Conv}\left(W \cdot \lambda^{*}\right)=-\operatorname{Conv}(W \cdot \lambda):=\Delta(\lambda) .
$$

Alexeev and Brion ([4]) and also Caldero ([8]) have constructed a flat deformation of the polarized flag variety $\left(X_{\lambda}, L_{\lambda}\right)$ to a polarized toric variety $\left(X_{\lambda ; 0}, L_{\lambda ; 0}\right)$ such that the moment polytope of $\left(X_{\lambda ; 0}, L_{\lambda ; 0}\right)$ is the string polytope $Q(\lambda)$.

Let $\Phi_{\lambda}: X_{\lambda ; 0} \rightarrow Q(\lambda) \subset \mathbb{R}^{\ell}$ be the moment map for $\mathbb{T}$-action, where $\mathbb{T}$ is the compact part of $\left(\mathbb{C}^{*}\right)^{\ell}$. Then the composition $\pi_{\lambda} \circ \Phi_{\lambda}: X_{\lambda ; 0} \rightarrow \Delta_{\lambda} \subset \Lambda_{\mathbb{R}}$ is the moment map for the maximal compact torus $T \subset H$.

Then apply the results from $\S 3$, we have

Theorem 4.1.1. The Chow quotient of $X_{\lambda}=G / P_{\lambda}$ by the maximal torus $H$ degenerates flatly to the Chow quotient of the toric variety $X_{\lambda ; 0}$ by the subtorus $H$. The corresponding fan of the toric Chow quotient is the common refinement of the normal fans of the polytopes $\pi_{\lambda}^{-1}(\mu) \cap Q(\lambda)$ for all $\mu \in \Delta(\lambda)$. In addition, with respect to some suitable ample line bundle, this toric Chow quotient corresponds to the fiber polytope $\Sigma\left(Q(\lambda), \Delta_{\lambda}\right)$ for the projection $\pi_{\lambda}: Q(\lambda) \rightarrow \Delta_{\lambda}$.

Proof. The flat family follows from Theorem 3.2.1. The rest is standard for Chow quotients of toric varieties and fiber polytopes (see [20] and [7]).

4.2. The toric variety $\bar{N}_{0, n}$. Let $\Delta_{2}^{n}$ be the second hypersymplex defined by

$$
\Delta_{2}^{n}=\left\{\left(r_{1}, \ldots, r_{n}\right) \mid 0 \leq r_{i} \leq 1, \sum_{i} r_{i}=2\right\}
$$

Any point $\left(r_{1}, \ldots, r_{n}\right) \in \Delta_{2}^{n}$ can be realized as side lengthes of a polygon in $\mathbb{R}^{3}$. Let $d_{2}, \ldots, d_{n-2}$ be the diagonals emanating from the start of the first edge to the ends of second to $(n-2)$-th edges. Then $\left(r_{1}, \ldots, r_{n}, d_{2}, \ldots, d_{n-2}\right)$ satisfies all possible triangle inequalities

$$
\begin{gathered}
d_{i}-d_{i+1} \leq r_{i+1}, \\
d_{i}-d_{i+1} \geq-r_{i+1}, \\
d_{i}+d_{i+1} \geq r_{i+1}
\end{gathered}
$$

for $1 \leq i \leq n-2$. Here $d_{1}=r_{1}$ and $d_{n-1}=r_{n}$. These inequalities define a polytope $\Gamma_{2}^{n}$ in $\mathbb{R}^{2 n-3}=\mathbb{R}^{n} \times R^{n-3}$, called a Gelfand-Tsetlin polytope (see, e.g., [12] for details). Let

$$
\varphi: \Gamma_{2}^{n} \rightarrow \Delta_{2}^{n}
$$


be the polytopal projection by forgetting the coordinates $d_{2}, \ldots, d_{n-2}$. Let $\Sigma\left(\Gamma_{2}^{n}, \Delta_{2}^{n}\right)$ be the fiber polytope of the projection $\varphi: \Gamma_{2}^{n} \rightarrow \Delta_{2}^{n}$ ([7]).

TheOREM 4.2.1. $\bar{M}_{0, n}$ admits a flat degeneration to the toric variety $\bar{N}_{0, n}$ defined by the fiber polytope $\Sigma\left(\Gamma_{2}^{n}, \Delta_{2}^{n}\right)$. The fan of $\bar{N}_{0, n}$ is the common refinement of the normal fans of all the polytopes $\varphi^{-1}\left(\left(r_{1}, \ldots, r_{n}\right)\right),\left(r_{1}, \ldots, r_{n}\right) \in \Delta_{2}^{n}$.

Proof. Take a flat family that degenerates $\operatorname{Gr}\left(2, \mathbb{C}^{n}\right)$ to the toric variety defined by $\Gamma_{2}^{n}$ (see, e.g., [8], [27] for the construction), then apply the results from $\S 3$ and [20]. D

We point it out again that $\bar{N}_{0, n}$ is sensitive to many choices involved. The one in the theorem is just a particular example of many toric degenerations of $\bar{M}_{0, n}$

Remark 4.2.2. In [21], Keel and Tevelev wrote down some equations for $\bar{M}_{0, n}$. Ideally, it would be nice if one can produce some binomials out of their equations, giving rise to certain toric degenerations of $\bar{M}_{0, n}$ with interesting binomial equations.

4.3. Moduli interpretations of $\bar{N}_{0, n}$. In some symplectic way, an interpretation can be done in terms of stable polygons ([14]). Topologically, this toric $\bar{M}_{0, n}$ can be obtained by collapsing the moduli spaces of stable polygons so that the bending flows extend everywhere ([17]). This is similar to Kamiyama and Yoshida's construction for the moduli space of ordinary polygons ([18]). But, we would be happier to have an algebro-geometric moduli interpretation.

Further details of these toric $\bar{N}_{0, n}$, including their moduli as well as polygonal interpretations, will appear in forthcoming papers ([17]).

Finally, unlike $\operatorname{Gr}\left(r, \mathbb{C}^{n}\right)$ with $r>2$, the Chow quotient of $\operatorname{Gr}\left(2, \mathbb{C}^{n}\right)$, namely $\bar{M}_{0, n}$, is nonsingular. This offers some hope that the total space of certain families in Corollary 3.2.3 may be chosen to be nonsingular as well. It will be essential for the future application to know the possible singularity of the total space of the degeneration. The author thanks the referee for suggesting this as a future research topic.

Acknowledgements. Many works have inspired this paper, in particular all those interesting papers on toric degenerations of flag varieties, Kapranov's paper that realizes $\bar{M}_{0, n}$ as Chow quotient, and Kapranov-Sturmfels-Zelevinsky's paper on (Chow) quotients of toric varieties. The paper of Alexeev and Brion ([4]) on toric deformation has been particularly helpful. I was convinced of toric degenerations of $\bar{M}_{0, n}$ for quite a while, I thank John Millson whose visit in the Spring of 2004 convinced me that it should be written up.

Thanks are also due to the referee for his/her useful comments and Diane Maclagan for her questions that lead to clarification and improvement.

\section{REFERENCES}

[1] V. Alexeev, Log canonical singularities and complete moduli of stable pairs, alggeom/9608013.

[2] V. Alexeev, Complete moduli in the presence of semiabelian group action, Ann. of Math. (2), 155 (2002), pp. 611-708.

[3] V. Alexeev, Higher-dimensional analogues of stable curves, math.AG/0607682.

[4] V. Alexeev AND M. BRION, Toric degenerations of spherical varieties, ArXiv: math.AG/0403379.

[5] V. Batyrev, I. Ciocan-Fontanine, B. Kim, D. van Straten, Mirror Symmetry and Toric Degenerations of Partial Flag Manifold, Acta Math., 184 (2000), pp. 1-39. 
[6] A. Berenstein and A. Zelevinsky, Tensor product multiplicities, canonical bases and totally positive variaties, Invent. Math., 143 (2001), pp. 77-128.

7] L. Bllera and B. Sturmfels, Fiber polytopes, Annals of Math., 135 (1992), pp. 527-549.

[8] P. Caldero, Toric degenerations of Schubert varieties, Transform. Groups, 7 (2002), pp. 51-60.

[9] P. Foth AND Y. Hu, Toric degenerations of weight varieties and applicationsmath, Travaux Mathématiques (2005), math.AG/0406329.

[10] P. HACKING, Compact moduli of hyperplane arrangements, math.AG/0310479.

[11] P. Hacking, S. Keel, And J. Tevelev, Compactification of the moduli space of hyperplane arrangements, math.AG/0501227.

[12] B. Howard, J. Millson, A. Snowden, and Ravi Vakil, The projective invariants of ordered points on the line, math.AG/0505096.

[13] B. Howard, J. Millson, A. Snowden, And Ravi Vakil, The moduli space of $n$ points on the line is cut out by simple quatrics when $n$ is not six, math.AG/0607372.

[14] Y. Hu, Moduli Spaces of Stable Polygons and Symplectic Structures on $\bar{M}_{0, n}$, Compositio Mathematica, 118 (1999), pp. 159-187.

[15] Y. Hu, Stable Configurations of Linear Subspaces and Vector Bundles, Quarterly Journal of Pure and Applied Mathematics, 1 (2005), pp. 127-164.

[16] Y. Hu, Topological Aspects of Chow Quotients, Journal of Differential Geometry, 69 (2005), pp. 399-440.

[17] Y. Hu, Toric $\bar{M}_{0, n}$ as Moduli Space, in preparation.

[18] Y. Kamiyama and T. Yoshida, Symplectic toric space associated to triangle inequalities, Geom. Dedicata, 93 (2002), pp. 25-36.

[19] M. Kapranov, Chow quotients of Grassmannian, I.M. Gelfand Seminar Collection, 29-110, Adv. Soviet Math., 16, Part 2, Amer. Math. Soc., Providence, RI, 1993.

[20] M. Kapranov, B. Sturmfels and A. Zelevinsky, Quotients of toric varieties, Math. Ann., 290 (1991), pp. 643-655.

[21] S. Keel and J. Tevelev, Equations for $\bar{M}_{0, n}$, math.AG/0507093.

[22] S. Keel And J. Tevelev, Geometry of Chow Quotients of Grassmannians, math.AG/0401159.

[23] M. Kogan and E. Miller, Toric degeneration of Schubert varieties and Gel'fand-Cetlin polytopes, math.AG/0303208.

[24] J. Kollár, Rational curves on algebraic varieties, Ergebnisse der Mathematik und ihrer Grenzgebiete. 3. Folge. A Series of Modern Surveys in Mathematics [Results in Mathematics and Related Areas. 3rd Series. A Series of Modern Surveys in Mathematics], 32. SpringerVerlag, Berlin, 1996.

[25] L. Lafforgue, Chirurgie des grassmanniennes, (French) [Surgery on Grassmannians] CRM Monograph Series, 19. American Mathematical Society, Providence, RI, 2003.

[26] L. Lafforgue, Pavages des simplexes, schémas de graphes recollés et compactification des $\mathrm{PGL}_{r}^{n+1} / \mathrm{PGL}_{r}$, Invent. Math., 136 (1999), pp. 233-271. Erratum: Invent. Math., 145 (2001), pp. 619-620.

[27] V. Lakshmibai and N. Gonciulea, Flag Varieties, Hermann 2001.

[28] D. Mumford, Geometric Invariant Theory, 1962, Berlin, New York. MR1304906, Zbl 0797.14004.

[29] L. Ness, Stratification of the null cone via the moment map, with an appendix by David Mumford, Amer. J. Math., 106 (1984), pp. 1281-1329, MR0765581.

[30] C. Teleman, Quantization Conjecture Revisted, Ann. of Math. 
Y. HU 